\title{
TRIM44 facilitates ovarian cancer proliferation, migration, and invasion by inhibiting FRK
}

\author{
Xiu-Zhang YU ${ }^{1,2}$, Jia-Ling YUAN ${ }^{1,2}$, Hui YE ${ }^{1,2}$, Ke YI ${ }^{1,2}$, Ming-Rong QIE ${ }^{1,2}$, Min-Min HOU ${ }^{1,2, *}$ \\ ${ }^{1}$ Department of Obstetrics and Gynecology, West China Second University Hospital, Sichuan University, Chengdu, Sichuan, China; ${ }^{2}$ Key Labora- \\ tory of Birth Defects and Related Diseases of Women and Children Sichuan University, Ministry of Education, Chengdu, Sichuan, China
}

${ }^{*}$ Correspondence: mminnh789@163.com

Received November 28, 2020 / Accepted March 3, 2021

\begin{abstract}
Ovarian cancer (OC) is the leading cause of gynecologic cancer-related death in the world. Accumulating evidence indicated the important role of TRIM44 in cancer development. However, how TRIM44 displays in OC and the underlying mechanism remained unclear. TRIM44 and FRK expression in OC tissues and cell lines were investigated by western blot and RT-qPCR. Histotype of tissue samples and patients' data were analyzed. Kaplan-Meier Curve was performed to validate the effect of TRIM44. Colony formation assay, MTT assay, Transwell assay, and wound-healing assay were applied to elucidate the function of TRIM44 in OC cells. CHIP assay was used to explore the association between TRIM44 and FRK. Finally, we performed SKOV3 xenografts in Balb/c nude mice to further confirm the involvement of TRIM44 in OC development. We found TRIM44 highly expressed while FRK displayed low expression in OC cell lines and tissues. Moreover, analysis of histotype of tissues and patients' data and Kaplan-Meier Curve implied the important role of TRIM44 and FRK in tumor progression. Further in vitro study suggested that knocking down TRIM44 inhibited OC cells proliferation, migration, and invasion. Besides, FRK was identified as the target gene of TRIM44 in OC, and TRIM44 promoted OC cells proliferation, migration, and invasion by inhibiting FRK. Finally, in vivo animal experiment further confirmed the promotive effect of TRIM44 on OC progression. Our findings demonstrated that TRIM44 facilitated OC proliferation, migration, and invasion by inhibiting FRK, providing new insights for theoretical research and therapy of OC.
\end{abstract}

Key words: TRIM44, ovarian cancer, FRK, proliferation, invasion

$\mathrm{OC}$ is the second common and the most fatal gynecologic cancer in the world [1-3]. Known as a silent killer, OC is difficult to be diagnosed until at an advanced stage. In fact, over $70 \%$ of cases are diagnosed at stage III or IV due to the asymptomatic nature of OC and lack of approaches for early diagnostics $[3,4]$. So far, the standard therapy for OC is debulking surgery combined with chemotherapy [4, 5]. However, the majority of patients will progress to recurrence and $75 \%$ of recurrence is incurable $[4,6]$. Although there are new approaches such as angiogenesis inhibitors and PARP inhibitors, these therapies are associated with added side effects or limited application $[4,7,8]$. Given that, it is promising to identify new targets and develop effective strategies for OC therapy.

The biological behavior of OC differs from hematogenous metastasis, which makes it easier to metastasize [9]. OC cells initially disseminate in the peritoneal cavity and only superficial cells are invasive $[9,10]$. However, owing to genetic and epigenetic factors, tumor cells process rapid proliferation and epithelial-to-mesenchymal transition (EMT) enables the cells to migrate and invade $[9,11]$. These cancer cells travel through peritoneal fluid and develop metastases [12, 13]. The high fatality rate of OC is mainly due to wide metastasis within the peritoneal cavity $[9,13]$. Thus, it is still essential to further uncover the mechanism of OC metastasis.

TRIM-containing protein 44 (TRIM44) is a member of the TRIM protein family, which contains a RING domain, a coiled-coil region, and one or two B boxes, and [14, 15]. TRIM family proteins have been found involved in varieties of processes including antiviral function, regulating innate immune reaction, and tumorigenesis [15-17]. Increasing evidence indicates the oncogenic role of TRIM44 in cancer development. Overexpression of TRIM44 facilitates cell proliferation, migration, and invasion in colorectal cancer (CRC), hepatocellular carcinoma, thyroid cancer, and non-small cell lung cancer (NSCLC) [18-21]. However, the function of TRIM44 in OC and the underlying mechanism still remains unknown. 
In this work, we performed experiments both in vitro and in vivo to investigate the function of TRIM44 in OC. Moreover, we explored the underlying mechanism that TRIM44 facilitated ovarian cancer progression by inhibiting FRK, providing new insights for theoretical research and therapy of OC.

\section{Materials and methods}

Tissue specimens. 15 primary ovarian cancer tissue samples and their paired 15 adjacent tissues were provided by West China Second University Hospital, Sichuan University. Written informed consents from patients were obtained before these clinical materials used for research purpose. This research was approved by the ethics committee of West China Second University Hospital, Sichuan University.

Cell culture and cell transfection. OC cell lines including SKOV3, OVCAR3, ES-2, A2780, COC1, and ovarian epithelial cell line HOSEpiC were obtained from the American Type Culture Collection (ATCC). Cells were cultured in DMEM (\#C12430500BT, Gibco) or RPMI-1640 (\#31800022, Gibco) with $10 \%$ fetal bovine serum (FBS) (\#10100147, Gibco) in a $5 \% \mathrm{CO}_{2}$ humidified incubator at $37^{\circ} \mathrm{C}$.

The overexpressing plasmids of FRK (pCDNA3.1FRK) and TRIM44 (pCDNA3.1-TRIM44) were conducted by cloning the corresponding full sequence into pCDNA3.1 (Addgene). The empty vector was used as a negative control (p-NC). shRNA for TRIM44 (sense: 5'-CCGGGGCTTGATTTGAGTACCTATTCTCGAGAATAGGTACTCAAATCAAGCCTTTTTG-3'; anti-sense: 5'-AATTCAAAAAGGCTTGATTTGAGTACCTATTCTCGAGAATAGGTACTCAAATCAAGCC-3') was inserted into PLKO.1 (Addgene). And PLKO.1 vector was used as a control (sh-NC). siRNA for FRK (5'-GGAGUACCUAGAACCCUAUTT-3') was synthesized by GenePharma (Shanghai, China). Scramble siRNA was used as a control (siNC). Lentiviral plasmids along with packaging plasmids were transfected into 293T cells via polyetherimide, and the virus supernatants were harvested 48-72 $\mathrm{h}$ after transfection. For lentiviral transfection, $1 \times 10^{5}$ cells were seeded in each well of 6-well plates and incubated at $37^{\circ} \mathrm{C}$ overnight. Appropriate lentiviral was added to the plate and medium was changed in $12-24 \mathrm{~h}$.

RNA isolation and RT-qPCR assays. Total RNA was extracted from cells or tissues by the TRIzol reagent (15596026, Invitrogen) according to the manufacturer's protocol. Complementary DNA was synthesized by reverse transcription kit (\#AT311-03, Transgene Biotech). RT-qPCR was conducted using SYBR Green Mix (\#172-5124, BIO-RAD) and the Applied Biosystems 7500 Real-Time PCR System. The relative mRNA level was calculated by the method of $2^{-}$ $\triangle \triangle C \mathrm{Ct}$. The following primers were used for RT-qPCR: TRIM44 forward: 5'-GTGGACATCCAAGAGGCAAT-3'; TRIM44 reverse: 5'-AGCAAGCCTTCATGTGTCCT-3'; FRK forward: 5'-ACCGCAACTCCATACAGC-3'; FRK reverse:
5'-TTCCGAGACTCCAGATAGGC-3'; FRK forward (CHIP): 5'-CACAGACAGAAAGAAACCAGGA-3'; FRK reverse (CHIP): 5'-TGAATAACCAACCAGCAGAGG-3'; GAPDH forward: 5'-AGGTCGGTGTGAACGGATTTG-3'; GAPDH reverse: 5'-GGGGTCGTTGATGGCAACA-3'.

Western blot. Cells or tissues were lysed in RIPA buffer and then were centrifuged at $12,000 \times \mathrm{g}$ at $4^{\circ} \mathrm{C}$ for $30 \mathrm{~min}$. An equal amount of protein was electrophoresed in SDS-PAGE gel and then transferred to polyvinylidene difluoride (PVDF) membranes. After milk blocking for an hour, the membranes were incubated with the TRIM44 antibody (ab236422, Abcam, 1:2000), FRK antibody (ab64914, Abcam, 1:1000), and $\beta$-actin (ab8827, Abcam, 1:2000) overnight at $4{ }^{\circ} \mathrm{C}$. After incubating with appropriate HRP-conjugated secondary antibodies for an hour, the signals were visualized by using enhanced chemiluminescence (ECL) and detected by AI600.

MTT assay. Thiazolyl blue tetrazolium bromide (MTT) was used to assess cell viability. 3000 cells were seeded in each well of 96-well plates and cultured in the incubator overnight. Each well of the plates was added with MTT reagent (\#C0009, Beyotime) and continued incubating for $4 \mathrm{~h}$ at $37^{\circ} \mathrm{C}$. Culture media was removed and $100 \mu \mathrm{L}$ DMSO (\#67685, Sigma) was added to dissolve the crystals (BS941, Biosharp). The absorbance was measured at $570 \mathrm{~nm}$.

Colony formation assay. A low density of 500-1000 cells/ well was seeded in 6-well plates and continued culturing for two weeks. Colonies were fixed in $4 \%$ paraformaldehyde followed by the staining with $0.5 \%$ crystal violet staining solution.

Wound healing assay. OC cells were seeded on 24-well plates and when the cells achieved full confluency, wounds were artificially scratched on cell monolayer by $200 \mu$ l pipette tips. The culture medium was discarded and then the cells were cultured in the medium without serum. Then wound healing photos were taken at the appropriate time, and the migrated cells were counted.

Transwell assay. Transwell chambers coated with matrigel (\#3422, Corning) were used to perform a Transwell assay. Briefly, the upper chambers were seeded with $5 \times 10^{4}$ cells suspended in medium without serum, and medium with $10 \%$ FBS was added onto the lower chambers. After incubation at $37^{\circ} \mathrm{C}$ for $24 \mathrm{~h}$, the cells were fixed in $4 \%$ paraformaldehyde and stained with $0.5 \%$ crystal violet. The number of invasive cells was counted on the microscope.

Chromatin immunoprecipitation (CHIP). EZ CHIP kit (EMD Millipore) was used to perform chromatin immunoprecipitation (CHIP). Briefly, formaldehyde was added to dishes and cells were cross-linked. Collected cells were sonicated on ice in an ultrasound bath, making the chromatin from 200 to $500 \mathrm{bp}$. Incubating with anti-IgG or anti-TRIM44 antibody overnight, immunoprecipitated DNA fragments were exacted using the kit. RT-qPCR was applied to detect the immunoprecipitated DNA fragments with primers for FRK. 
Animal experiment. Balb/c nude mice (4-5-week-old) were divided into sh-NC group and sh-TRIM44 group randomly. Established SKOV3 cells (sh-NC and sh-TRIM44) were subcutaneously inoculated into mice of the two groups. Tumor growth was observed and recorded every 5 days. 30 days after inoculation, the mice were sacrificed and tumors were surgically dissected and subjected to western blot and RT-qPCR. The animal study was performed in accordance with the institutional guidelines for the care and use of laboratory animals.

Statistical analysis. GraphPad Prism 6 software was used to perform statistical analysis. All data were presented as the means \pm SD from 3 independent experiments. Student's t-test or ANOVA with Tukey's post-hoc test was used to determine the difference between groups. Results were considered statistically significant when $\mathrm{p}<0.05$.

\section{Results}

TRIM44 and FRK are abnormally expressed in OC cell lines and tissues. To explore the potential role of TRIM44 and FRK in OC development, we first investigated TRIM44 and FRK expression in 15 primary OC specimens and their paired 15 adjacent tissues by RT-qPCR and western blot. Compared to normal tissues, TRIM44 expression was upregulated while FRK expression was downregulated in OC tissues (Figures 1A, 1B). We next examined TRIM44 and FRK expression in OC cell lines. In accordance with the results in tissues, high expression of TRIM44 and low expression of FRK were observed in OC cell lines compared with normal ovarian HOSEpiC cells (Figures 1C, 1D). We analyzed the histotype of 15 tissue samples and patients' data. Results showed that high expression of TRIM44 was significantly correlated with advanced TNM stage, larger tumor size, and lymph node metastasis, while it was not related to the patients' age. And the low expression of FRK was significantly related to advanced TNM stage and lymph node metastasis while the tumor size and patients' age were not related (Figures 1E, 1F). More than that, high expression of TRIM44 was correlated with a lower three-year survival rate and low expression of FRK was correlated with a higher three-year survival rate, implying the important role of TRIM44 and FRK in tumor progression (Figures 1G, 1H). These results indicated that TRIM44 and FRK might be involved in OC development.

Knocking down TRIM44 inhibits OC cells' proliferation, migration, and invasion. To elucidate the exact function of TRIM44 in OC progression, we overexpressed or silenced TRIM44 by lentiviral transfection and examined its effect on OC cells' proliferation, migration, and invasion. TRIM44 expression was successfully inhibited in the sh-TRIM44 group and significantly increased in the p-TRIM44 group (Figure 2A). As shown in Figures 2B-2E, silencing TRIM44 significantly inhibited cell viability, proliferation, migration, and invasion, while the remarkable promotion was observed in the group of TRIM44 overexpression in both SKOV3 and OVCAR3 cell lines. All these data suggested silencing TRIM44 inhibits OC cells' proliferation, migration, and invasion.

FRK is a target gene of TRIM44 in OC. As shown in Figure 1, TRIM44 expression and FRK expression were negatively correlated to some extent, so we were interested to figure out the direct association between TRIM44 and FRK. It was reported TRIM44 might target FRK in renal cell carcinoma [22], so we performed a CHIP assay to detect the interaction between TRIM44 and FRK in SKOV3 and OVCAR3 cells. Results showed that TRIM44 could directly bind to the FRK gene both in SKOV3 and OVCAR3 cell lines (Figure 3A), suggesting FRK might be a target gene of TRIM44 in OC cells. Additionally, inhibition of TRIM44 significantly promoted FRK expression, while overexpressing TRIM44 inhibited FRK expression (Figures 3B, 3C). These results implied that TRIM44 targeted FRK in SKOV3 and OVCAR3 cells.

TRIM44 promotes OC cells' proliferation, migration, and invasion by inhibiting FRK. Furthermore, we wanted to explore whether TRIM44 facilitating OC development was related to FRK. Compared to the p-NC group, FRK expression was remarkably increased in the p-FRK group (Figure 4A). FRK overexpression notably inhibited OC cells viability, proliferation, migration, and invasion (Figures 4B-4E). However, when co-overexpressed with TRIM44, the inhibition caused by FRK was reversed (Figures $4 \mathrm{~B}-4 \mathrm{E}$ ). Altogether, these data suggested that TRIM44 promoted OC cells' proliferation, migration, and invasion by inhibiting FRK.

Knocking down TRIM44 inhibits SKOV3 tumor growth. In view of the in vitro results, we next investigated the in vivo effect of TRIM44 in SKOV3 xenografts. Knocking down TRIM44 significantly inhibited the tumor formation (Figures 5A, 5B), and the average tumor weight in the sh-TRIM44 group was much lower than in the control group (Figure 5C), indicating TRIM44 silencing inhibited SKOV3 tumor growth in vivo. TRIM44 and FRK expression were further investigated in the tumor tissues and the result indicated that TRIM44 expression was inhibited while FRK expression was notably increased in the sh-TRIM44 group (Figures 5D, 5E). These results further demonstrated TRIM44 promoted OC development by inhibiting FRK.

\section{Discussion}

OC is the leading cause of gynecologic diseases related death [1]. Despite the notable advances in OC, current therapy still mainly depends on surgery as well as combined therapy [4]. Furthermore, about $25 \%$ of patients will develop chemoresistance and the majority ultimately experience recurrence $[4,13]$. In this research, we observed TRIM44 was highly expressed either in OC cell lines or OC tissues, and analysis of histotype of tissues and patients' data and KaplanMeier Curve demonstrated that high TRIM44 expression and low FRK expression were correlated with tumor progres- 

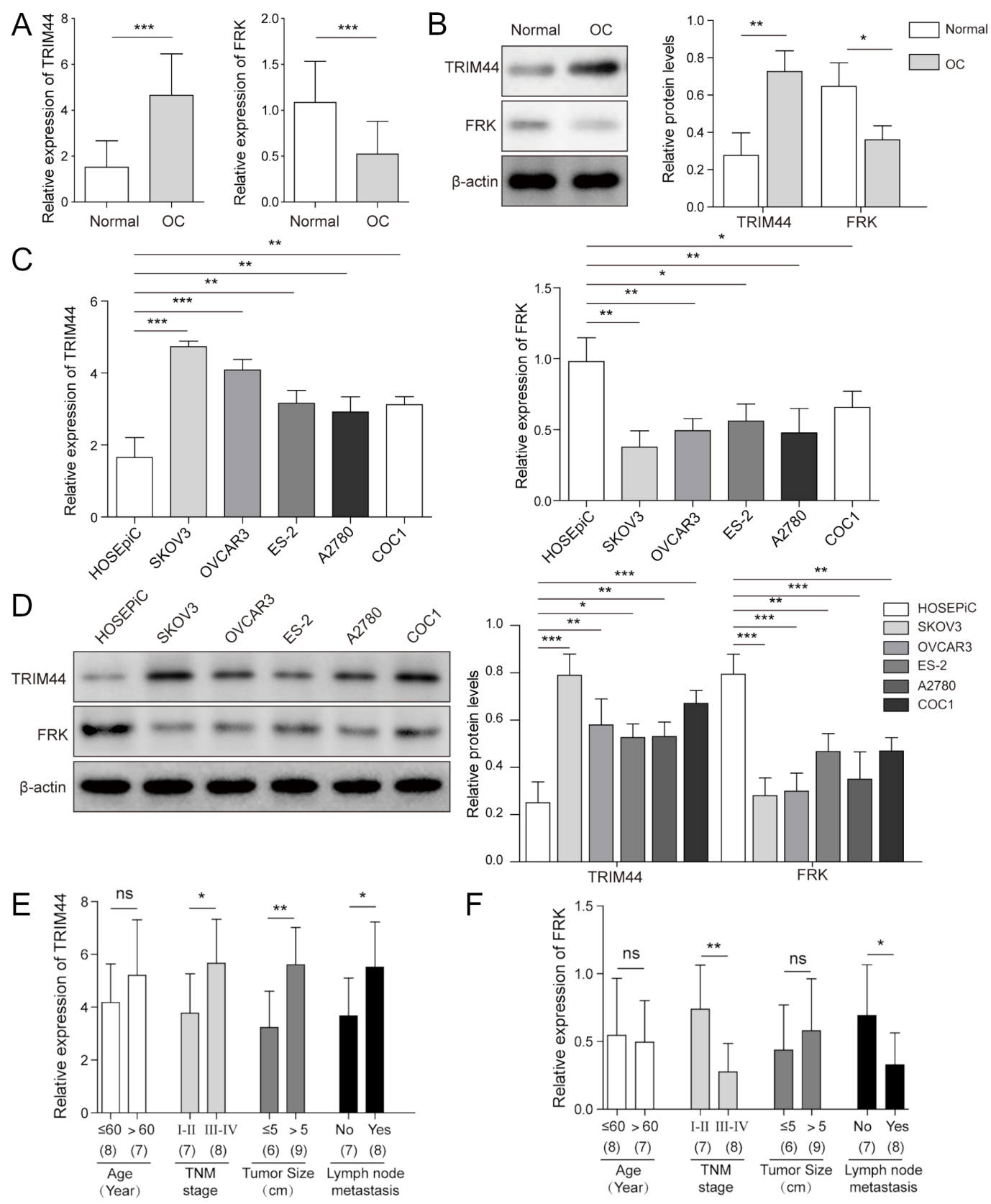

$\mathrm{F}$

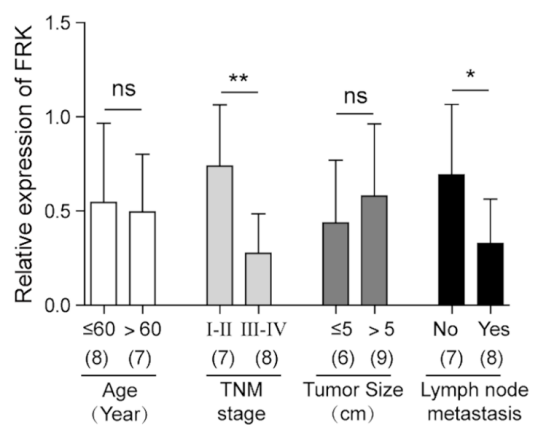

G

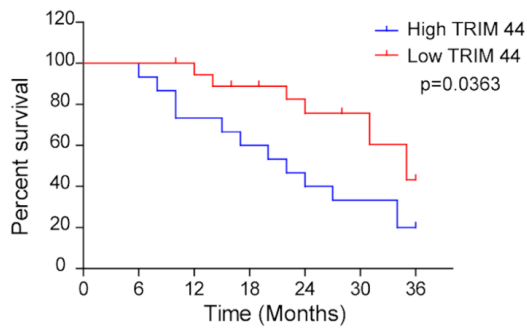

$\mathrm{H}$

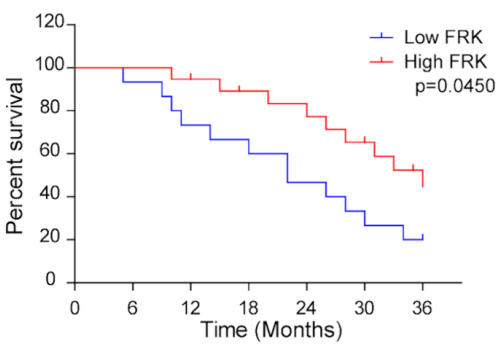

Figure 1. TRIM44 and FRK are abnormally expressed in OC cell lines and tissues. A) RT-qPCR assay was performed to detect the relative mRNA level of TRIM44 and FRK in OC tissues ( $(n=15)$. B) Relative protein expressions of TRIM44 and FRK in OC tissues were investigated by western blot ( $n=15)$. C) RT-qPCR was performed to detect the relative mRNA level of TRIM44 and FRK in OC cell lines. D) Western blot was used to investigate the relative protein level of TRIM44 and FRK in OC cell lines. E, F) RT-qPCR assay was used to detect the relative mRNA level of TRIM44 and FRK in OC tissues $(n=15)$, and histotype and patients' data were analyzed. $(\mathrm{G}-\mathrm{H})$ Kaplan-Meier Curve of TRIM44 expression and OC patient survival $(\mathrm{n}=50) .{ }^{*} \mathrm{p}<0.05$, ${ }^{* *} \mathrm{p}<0.01,{ }^{* * *} \mathrm{p}<0.001$ 

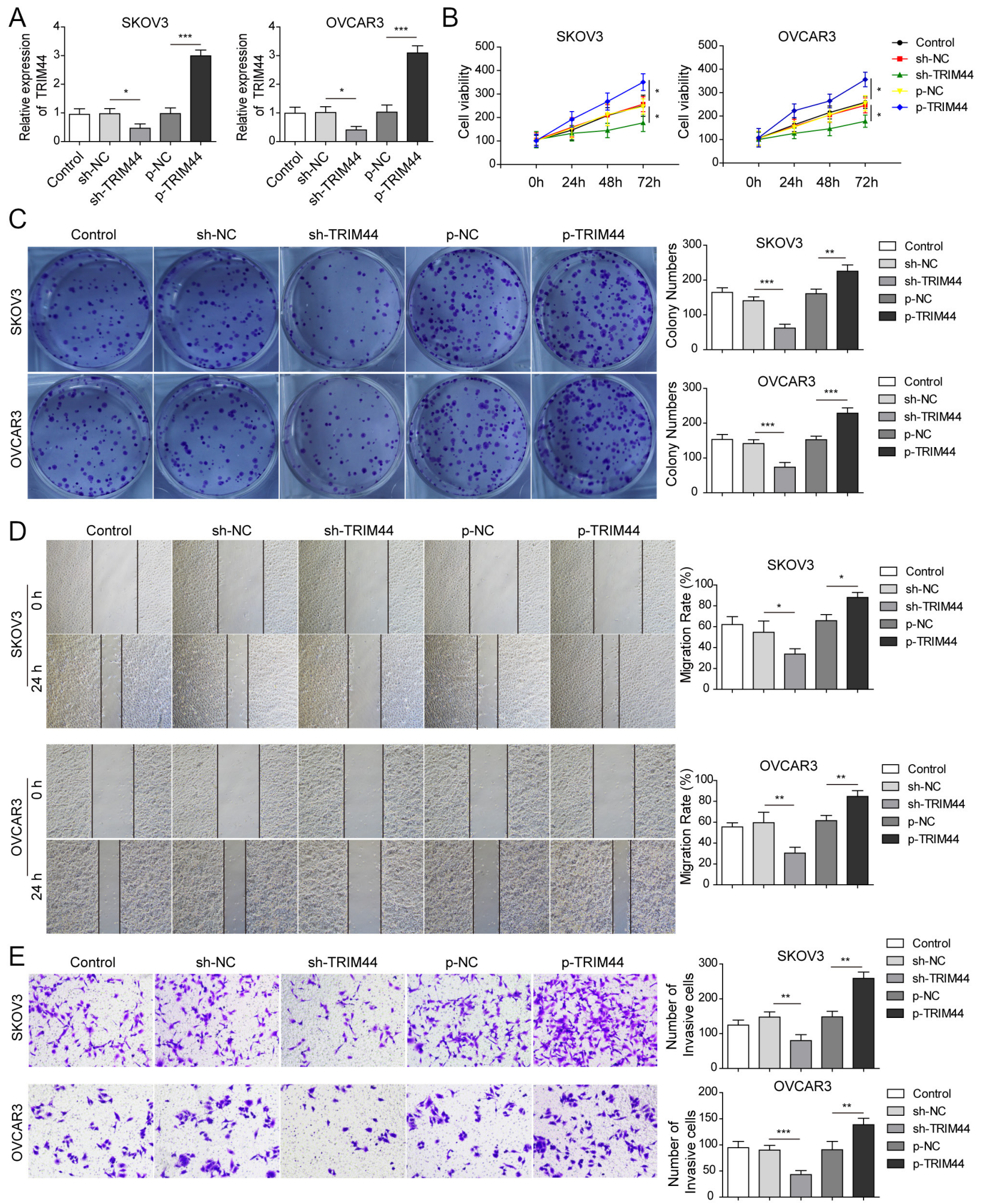

Figure 2. Knocking down TRIM44 inhibits OC cells' proliferation, migration, and invasion. A) TRIM44 expression was manipulated by p-TRIM44 and shTRIM44, while p-NC and sh-NC were used as control, respectively. SKOV3 and OVCAR3 cells were transfected with sh-NC, sh-TRIM44, p-NC, and p-TRIM44, and relative mRNA expression of TRIM44 was detected by RT-qPCR. B) MTT assay was used to detect cell viability. C) Cell proliferation was investigated by conducting a colony formation assay. D) Wound-healing assay was performed to investigate cell migration. E) Transwell assay was performed to inspect cell invasion. ${ }^{*} \mathrm{p}<0.05,{ }^{* *} \mathrm{p}<0.01,{ }^{* * *} \mathrm{p}<0.001$ 

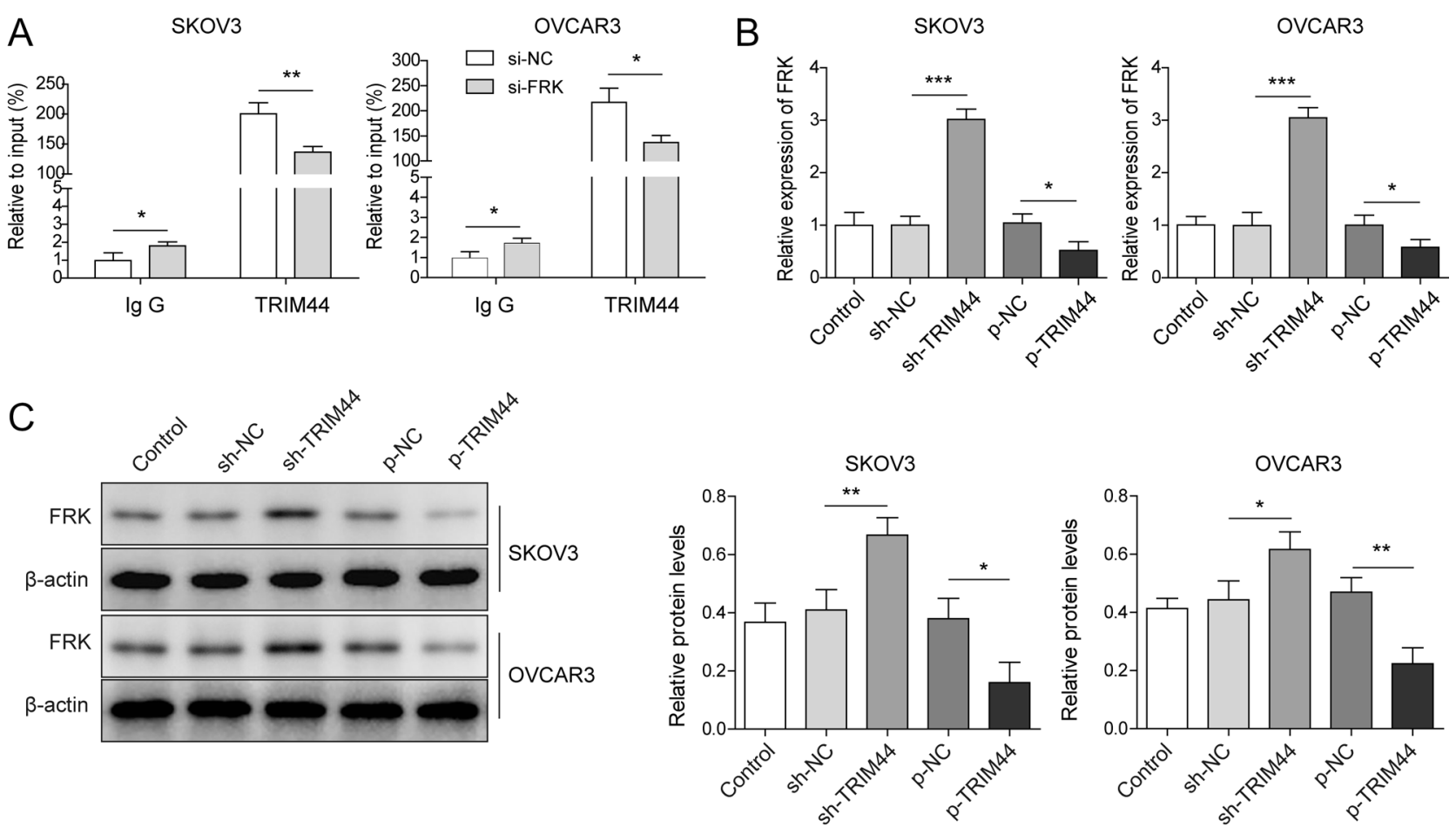

Figure 3. FRK is a target gene of TRIM44 in OC. A) FRK expression was manipulated by si-FRK and si-NC was used as a negative control. SKOV3 and OVCAR3 cells were transfected with si-NC and si-FRK, and cell lysates were used for the ChIP assay by using anti-IgG and anti-TRIM44 antibodies. B) sh-NC, sh-TRIM44, p-NC, and p-TRIM44 were transfected into SKOV3 and OVCAR3 cells, and RT-qPCR was performed to detect the relative mRNA expression of FRK. C) sh-NC, sh-TRIM44, p-NC, and p-TRIM44 were transfected into SKOV3 and OVCAR3 cells, and to detect the relative protein expression of FRK, western blot was performed. ${ }^{*} \mathrm{p}<0.05,{ }^{* *} \mathrm{p}<0.01$, ${ }^{\star * *} \mathrm{p}<0.001$

sion. Silencing TRIM44 significantly inhibited OC development and malignant progression both in vitro and in vivo, indicating the oncogenic role of TRIM44 in OC. Furthermore, we demonstrated FRK was a target gene of TRIM44 in OC, and TRIM44 promoted OC proliferation, migration, and invasion by inhibiting FRK.

Accumulating evidence has demonstrated that TRIM44 participated in a variety of cancer development and displayed an oncogenic role $[19,20,23,24]$. TRIM44 overexpression contributed to cell proliferation, migration, and invasion in lung cancer, colorectal cancer, and prostate cancer [18, 21, 25]. Additionally, Liu et al. reported that TRIM44 could be used as a biomarker, which is valuable for clinical diagnosis and prognosis of cervical cancer [26]. A previous study has indicated that TRIM44 displayed high expression in epithelial ovarian cancer (EOC) tissues and low expression in the normal ovarian tissues, and was collected with the prognosis of EOC [27]. However, the exact function and underlying mechanism remain unstated. In this research, we further confirmed that TRIM44 was highly expressed either in OC cell lines or OC tissues, in contrast to the normal control. An analysis of patients' data further showed high TRIM44 expression was related to advanced TNM stage, larger tumor size, and lower three-year survival rate. Besides, we found
TRIM44 contributed to OC cell proliferation, migration, and invasion. Moreover, in vivo SKOV3 xenografts revealed silencing TRIM44 inhibited tumor formation and growth. All these data suggest TRIM44 plays an oncogenic role in $\mathrm{OC}$, providing a potential target for OC therapy.

The fyn-related kinase (FRK) belongs to the BRK family kinases (BFKs), which is originally called RAK [28, 29]. Since it was identified in breast tissues and cells in 1993 [30], varieties of studies have revealed its involvement in diverse tumor progression [28]. FRK could function as a tumor promoter or tumor suppressor in different cancers [28]. Craven et al. have indicated FRK suppressed colony formation in NIH3T3 cells [31]. FRK inhibited tumorigenesis and progression in breast cancer, glioma, and lung cancer [32-35]. Apart from the highlighted function of the tumor suppressor, FRK also could promote cell growth and invasion in liver cancer, displaying as an oncogene $[36,37]$. Yuta et al. reported TRIM44 facilitated renal cell carcinoma proliferation and migration via inhibiting FRK, suggesting FRK as a target gene in renal cell carcinoma [22]. However, an association between TRIM44 and FRK in OC remains poorly investigated. In this research, low expression of FRK was observed in OC tissues and cell lines, negatively correlated with TRIM44 expression. Additionally, by analyzing clinical samples, low FRK expres- 

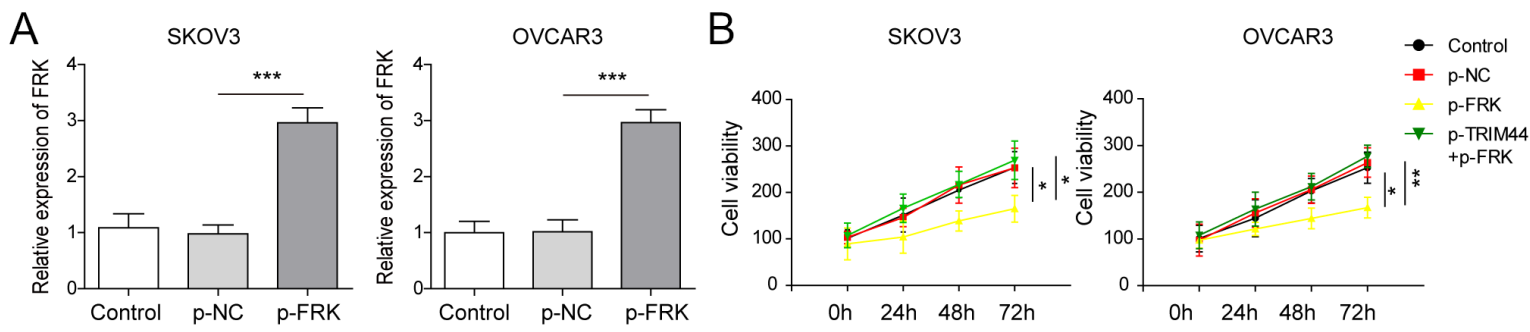

C Control p-NC p-FRK

p-TRIM44+p-FRK
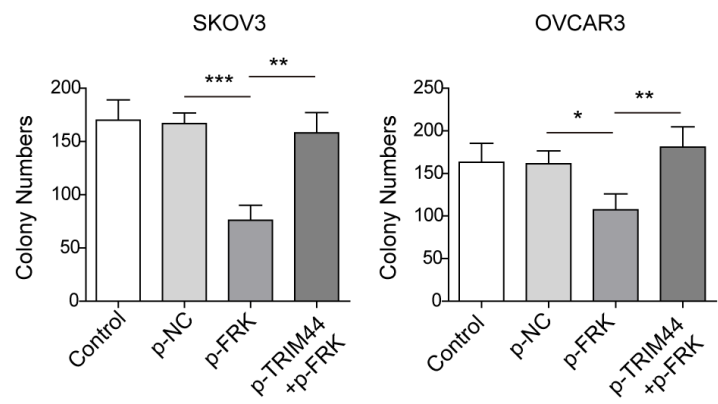

D
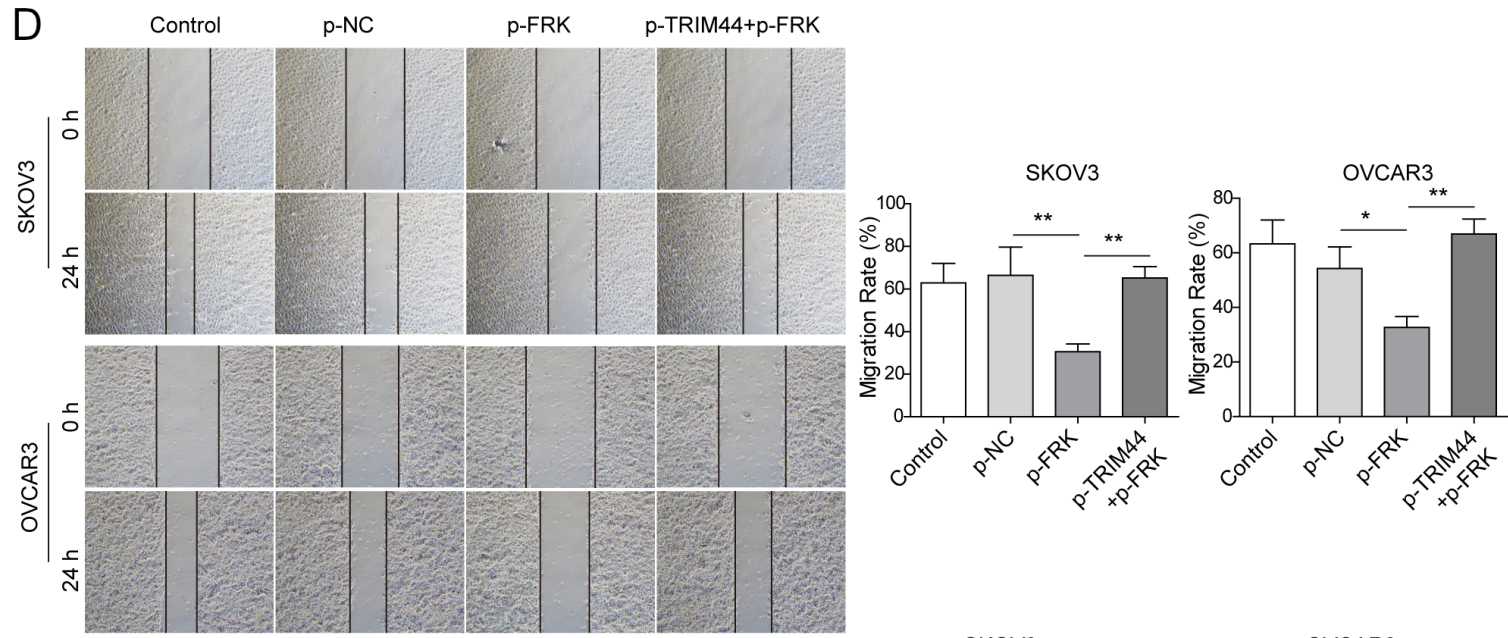

E
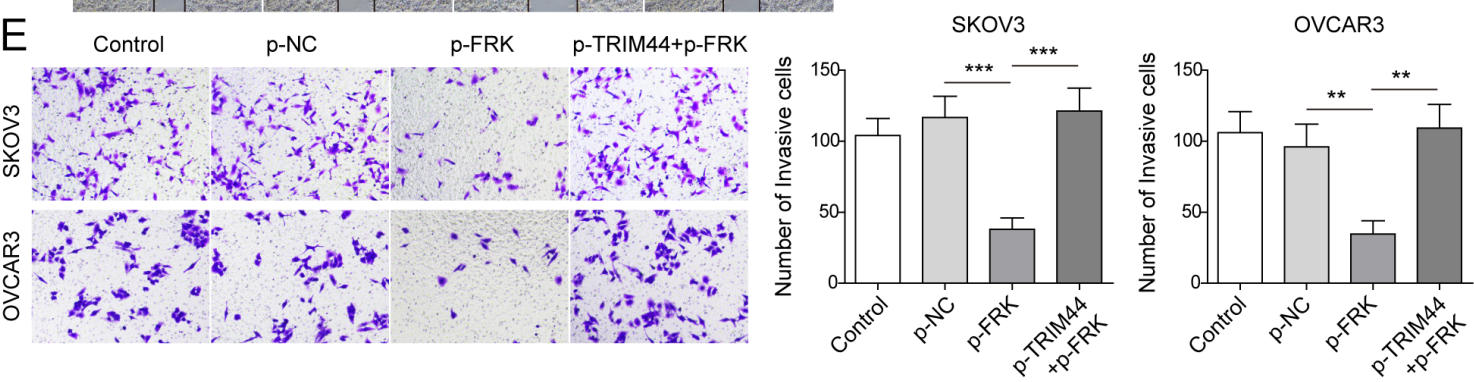

Figure 4. TRIM44 promotes OC cells' proliferation, migration, and invasion by inhibiting FRK. A) SKOV3 and OVCAR3 cells were plated followed by transfection with p-NC and p-FRK, and RT-qPCR was then applied to detect the FRK mRNA level. B) MTT assay was performed to investigate cell viability. C) In order to investigate cell proliferation, a colony formation assay was performed. $D$ ) Wound-healing assay was performed to investigate cell migration. E) Transwell assay was performed to investigate cell invasion. ${ }^{\star} p<0.05,{ }^{* *} p<0.01,{ }^{\star * *} p<0.001$

sion was found to correlate with advanced TNM stage and lower three-year survival rate. Moreover, FRK was identified to be the target gene of TRIM44 in OC. And FRK overexpression could facilitate OC cells' proliferation, migration, and invasion, while co-expressed with TRIM44 reversed the effect. Our study demonstrated the FRK played a tumor suppressor role and TRIM44 facilitated OC cells proliferation, migration, and invasion via inhibiting FRK. 

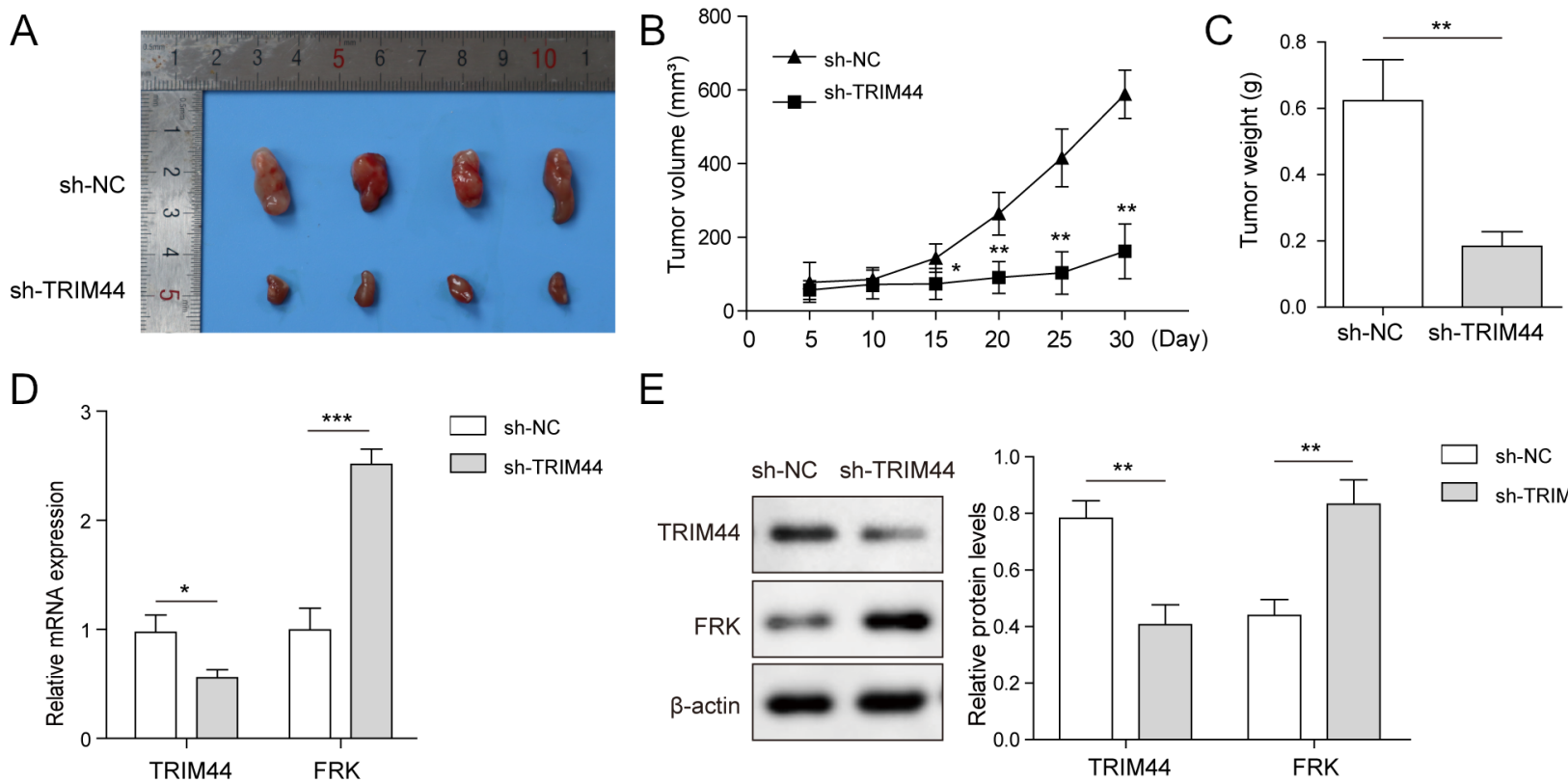

$E$
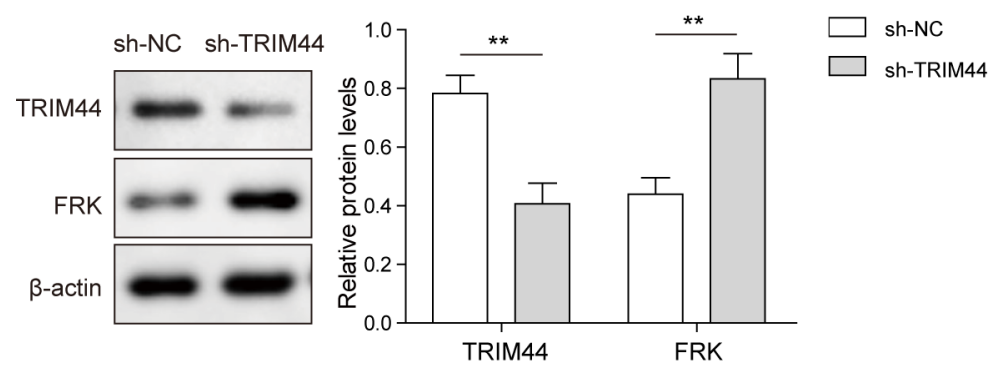

Figure 5. Knocking down TRIM44 inhibits SKOV3 tumor growth in vivo. A) Photographs of tumors from groups of sh-NC and sh-TRIM44. B) Tumor growth curves of each group. C) Tumors were weighed and analyzed, $n=4$ per group. D) Relative mRNA expression of TRIM44 and FRK in tumor tissues was measured by RT-qPCR. E) Western blot was conducted to examine the relative protein level of TRIM44 and FRK in the tumor. ${ }^{*} \mathbf{p}<0.05$, ${ }^{* *} \mathrm{p}<0.01,{ }^{* * *} \mathrm{p}<0.001$

In conclusion, this study indicated TRIM44 promoted OC cells' proliferation, migration, and invasion, which was regulated by inhibiting FRK, providing new insights and potential strategy for OC treatment and prognosis.

Acknowledgments: We would like to give our sincere gratitude to the reviewers for their constructive comments.

\section{References}

[1] REID BM, PERMUTH JB, SELLERS TA. Epidemiology of ovarian cancer: a review. Cancer Biol Med 2017; 14: 9-32. https://doi.org/10.20892/j.issn.2095-3941.2016.0084

[2] LHEUREUX S, GOURLEY C, VERGOTE I, OZA AM. Epithelial ovarian cancer. Lancet 2019; 393: 1240-1253. https:// doi.org/10.1016/S0140-6736(18)32552-2

[3] STEWART C, RALYEA C, LOCKWOOD S. Ovarian Cancer: An Integrated Review. Semin Oncol Nurs 2019; 35: 151156. https://doi.org/10.1016/j.soncn.2019.02.001

[4] CORTEZ AJ, TUDREJ P, KUJAWA KA, LISOWSKA KM. Advances in ovarian cancer therapy. Cancer Chemother Pharmacol 2018; 81: 17-38. https://doi.org/10.1007/s00280017-3501-8

[5] VERGote I, AMANT F, KRISTENSEN G, EHLEN T, REED NS et al. Primary surgery or neoadjuvant chemotherapy followed by interval debulking surgery in advanced ovarian cancer. Eur J Cancer 2011; 47: S88-92. https://doi. org/10.1016/S0959-8049(11)70152-6
[6] LOPEZ-GUERRERO JA, ROMERO I, POVEDA A. Trabectedin therapy as an emerging treatment strategy for recurrent platinum-sensitive ovarian cancer. Chin J Cancer 2015; 34: 41-49. https://doi.org/10.5732/cjc.014.10278

[7] FRANZESE E, CENTONZE S, DIANA A, CARLINO F, GUERRERA LP et al. Cancer Treat Rev 2019; 73: 1-9. https://doi.org/10.1016/j.ctrv.2018.12.002

[8] ARAVANTINOS G, PECTASIDES D. Bevacizumab in combination with chemotherapy for the treatment of advanced ovarian cancer: a systematic review. J Ovarian Res 2014; 7: 57. https://doi.org/10.1186/1757-2215-7-57

[9] LENGYEL E. Ovarian cancer development and metastasis. Am J Pathol 2010; 177: 1053-1064. https://doi.org/10.2353/ ajpath.2010.100105

[10] TARIN D, PRICE JE, KETTLEWELL MG, SOUTER RG, VASS AC et al. Mechanisms of human tumor metastasis studied in patients with peritoneovenous shunts. Cancer Res 1984; 44: 3584-3592.

[11] BRABLETZ T, KALLURI R, NIETO MA, WEINBERG RA. EMT in cancer. Nat Rev Cancer 2018; 18: 128-134. https:// doi.org/10.1038/nrc.2017.118

[12] SHIELD K, RILEY C, QUINN MA, RICE GE, ACKLAND ML et al. Alpha2beta1 integrin affects metastatic potential of ovarian carcinoma spheroids by supporting disaggregation and proteolysis. J Carcinog 2007; 6: 11. https://doi. org/10.1186/1477-3163-6-11

[13] NAORA H, MONTELL DJ. Ovarian cancer metastasis: integrating insights from disparate model organisms. Nat Rev Cancer 2005; 5: 355-366. https://doi.org/10.1038/nrc1611 
[14] URANO T, USUI T, TAKEDA S, IKEDA K, OKADA A et al. TRIM44 interacts with and stabilizes terf, a TRIM ubiquitin E3 ligase. Biochem Biophys Res Commun 2009; 383: 263-268. https://doi.org/10.1016/j.bbrc.2009.04.010

[15] KASHIMOTO K, KOMATSU S, ICHIKAWA D, ARITA T, KONISHI $\mathrm{H}$ et al. Overexpression of TRIM44 contributes to malignant outcome in gastric carcinoma. Cancer Sci 2012; 103: 2021-2026. https://doi.org/10.1111/j.13497006.2012.02407.x

[16] YANG B, WANG J, WANG Y, ZHOU H, WU X et al. Novel Function of Trim44 Promotes an Antiviral Response by Stabilizing VISA. J Immunol 2013; 190: 3613-3619. https://doi. org/10.4049/jimmunol.1202507

[17] MCNAB FW, RAJSBAUM R, STOYE JP, O'GARRA A. Tripartite-motif proteins and innate immune regulation. Curr Opin Immunol 2011; 23: 46-56. https://doi.org/10.1016/j. coi.2010.10.021

[18] LI CG, HU H, YANG XJ, HUANG CQ, YU XQ. TRIM44 Promotes Colorectal Cancer Proliferation, Migration, and Invasion Through the Akt/mTOR Signaling Pathway. Onco Targets Ther 2019; 12: 10693-10701. https://doi.org/10.2147/ OTT.S228637

[19] ZHOU Z, LIU Y, MA M, CHANG L. Knockdown of TRIM44 inhibits the proliferation and invasion in papillary thyroid cancer cells through suppressing the Wnt/ $\beta$-catenin signaling pathway. Biomed Pharmacother 2017; 96: 98-103. https://doi.org/10.1016/j.biopha.2017.09.132

[20] ZHU X, WU Y, MIAO X, LI C, YIN H et al. High expression of TRIM44 is associated with enhanced cell proliferation, migration, invasion, and resistance to doxorubicin in hepatocellular carcinoma. Tumour Biol 2016; 37: 14615-14628. https://doi.org/10.1007/s13277-016-5316-3

[21] LUO Q, LIN H, YE X, HUANG J, LU S et al. Trim44 facilitates the migration and invasion of human lung cancer cells via the NF-kappa B signaling pathway. Int J Clin Oncol 2015; 20: 508-517. https://doi.org/10.1007/s10147-014-0752-9

[22] YAMADA Y, KIMURA N, TAKAYAMA KI, SATO Y, SUZUKI T et al. TRIM44 promotes cell proliferation and migration by inhibiting FRK in renal cell carcinoma. Cancer Sci 2020; 111: 881-890. https://doi.org/10.1111/cas.14295

[23] WEI CY, WANG L, ZHU MX, DENG XY, WANG DH et al. TRIM44 activates the AKT/mTOR signal pathway to induce melanoma progression by stabilizing TLR4. J Exp Clin Cancer Res 2019; 38: 137. https://doi.org/10.1186/s13046-0191138-7

[24] KAWABATA H, AZUMA K, IKEDA K, SUGITANI I, KINOWAKI K et al. TRIM44 Is a Poor Prognostic Factor for Breast Cancer Patients as a Modulator of NF- $\mathrm{kB}$ Signaling. Int J Mol Sci 2017; 18: 1931. https://doi.org/10.3390/ ijms18091931

[25] TAN Y, YAO H, HU J, LIU L. Knockdown of TRIM44 Inhibits the Proliferation and Invasion; in Prostate Cancer Cells. Oncol Res 2017; 25: 1253-1259. https://doi.org/10.3727/096 504017X14854310794561
[26] LIU S, MENG F, DING J, JI H, LIN M et al. High TRIM44 expression as a valuable biomarker for diagnosis and prognosis in cervical cancer. Biosci Rep 2019; 39: BSR20181639. https://doi.org/10.1042/BSR20181639

[27] LIU S, YIN H, JI H, ZHU J, MA R. Overexpression of TRIM44 is an independent marker for predicting poor prognosis in epithelial ovarian. Exp Ther Med 2018; 16: 30343040. https://doi.org/10.3892/etm.2018.6541

[28] GOEL RK, LUKONG KE. Understanding the cellular roles of Fyn-related kinase (FRK): implications in cancer biology. Cancer Metastasis Rev 2016; 35: 179-199. https://doi. org/10.1007/s10555-016-9623-3

[29] BRAUER PM, TYNER AL. RAKing in AKT: A tumor suppressor function for the intracellular tyrosine kinase FRK. Cell Cycle 2009; 8: 2728-2732. https://doi.org/10.4161/ cc.8.17.9389

[30] CANCE WG, CRAVEN RJ, WEINER TM, LIU ET. Novel protein kinases expressed in human breast cancer. Int J Cancer 1993; 54: 571-577. https://doi.org/10.1002/ ijc.2910540409

[31] CRAVEN RJ, CANCE WG, LIU ET. The nuclear tyrosine kinase Rak associates with the retinoblastoma protein $\mathrm{pRb}$. Cancer Res 1995; 55: 3969-3972.

[32] BERCLAZ G, ALTERMATT HJR, ROHRBACH V, DREHER E, ZIEMIECKI A et al. Hormone-dependent nuclear localization of the tyrosine kinase iyk in the normal human breast epithelium and loss of expression during carcinogenesis. Int J Cancer 2000; 85: 889-894. https://doi.org/10.1002/ (sici)1097-0215(20000315)85:6<889::aid-ijc25>3.0.co;2-4

[33] HUA L, ZHU M, SONG X, WANG J, FANG Z et al. FRK suppresses the proliferation of human glioma cells by inhibiting cyclin D1 nuclear accumulation. J Neurooncol 2014; 119: 49-58. https://doi.org/10.1007/s11060-014-1461-y

[34] SUN B, YANG N, JIANG Y, ZHANG H, HOU C et al. Antagomir-1290 suppresses CD133 cells in non-small cell lung cancer by targeting fyn-related Src family tyrosine kinase. Tumour Biol 2015; 36: 6223-6230. https://doi.org/10.1007/ s13277-015-3307-4

[35] OGUNBOLUDE Y, DAI C, BAGU ET, GOEL RK, LUKONG KE. FRK inhibits breast cancer cell migration and invasion by suppressing Epithelial-mesenchymal transition. Oncotarget 2017; 8: 113034-113065. https://doi.org/10.18632/oncotarget. 22958

[36] CHEN JS, HUNG WS, CHAN HH, TSAI SJ, SUN HS. In silico identification of oncogenic potential of fyn-related kinase in hepatocellular carcinoma. Bioinformatics 2013; 29: 420-427. https://doi.org/10.1093/bioinformatics/bts715

[37] PILATI C, LETOUZE E, NAULT JC, IMBEAUD S, BOULAI A et al. Genomic profiling of hepatocellular adenomas reveals recurrent FRK-activating mutations and the mechanisms of malignant transformation. Cancer Cell 2014; 25: 428-441. https://doi.org/10.1016/j.ccr.2014.03.005 